\title{
SIMULAÇÃO DA PRODUTIVIDADE DE BATATA EM FUNÇÃO DA REGULAGEM DO ASPERSOR (SISTEMA PORTÁTIL)
}

\author{
Rubens Duarte Coelho', Marcos Vinícius Folegatti² e José Antônio Frizzone ${ }^{2}$
}

\begin{abstract}
RESUMO
O presente trabalho diz respeito a um estudo realizado em laboratório, com modelo de aspersor rotativo de impacto de pequeno porte, visando quantificar a influência da pressão de operação, da velocidade de rotação e do período de oscilação do defletor-martelo, na uniformidade de distribuição de água, de maneira tal a simular, em computador, a produtividade da cultura da batata (função de produção) em diferentes pontos de regulagem do aspersor. Nos ensaios de distribuição espacial das lâminas d'água, utilizou-se a metodologia de distribuição radial de coletores, combinando-se três pressões de operação $(200,300$ e 400kPa), cinco tempos de rotação do aspersor $(7,15,30,60$ e 90 segundos) e quatro níveis de tensão da mola acoplada ao defletor-martelo (ângulos de deflexão $0^{\circ}$, $60^{\circ}, 240^{\circ}$ e $480^{\circ}$ ). Com os resultados obtidos de coeficiente de uniformidade de Christiansen e com a produção da cultura da batata para espaçamentos entre aspersores de $6 \times 12,12 \times 12,12 \times 15$, $12 \times 18,18 \times 18,18 \times 24$ e $24 \times 24 \mathrm{~m}$, efetuou-se uma análise multifatorial de variância pelo método do intervalo de confiança. Observou-se que os maiores tempos de rotação dos aspersores apresentaram melhores coeficientes de uniformidade nos espaçamentos-limite recomendados pelo fabricante, e que o ângulo de deflexão de $240^{\circ}$ da mola acoplada ao defletor-martelo é o limite máximo de deflexão para que não ocorra interferência prejudicial à distribuição espacial da água.
\end{abstract}

Palavras-chave: irrigação, aspersores, espaçamentos, regulagem

\section{SIMULATION OF POTATO YIELD AS A FUNCTION OF SPRINKLER ADJUSTMENT IN A PORTABLE SYSTEM}

\begin{abstract}
The present study was carried out with a rotary irrigation sprinkler with the objective of quantifying the influence of rotation rate, operational pressure and oscillation period of the impact arm coupled to the sprinkler, on the spatial distribution of water, in such a way as to simulate, through production functions, the potato crop production under portable irrigation systems. The tests were conducted with the combination of three factors: operational pressure $(200,300$, and $400 \mathrm{kPa})$, time for a complete rotation $(7,15,30,60$ and $90 \mathrm{sec}$.) and the adjustment level of the spring tension coupled to the impact arm (deflection angle $0^{\circ}, 60^{\circ}, 240^{\circ}$ e $480^{\circ}$ ). With the coefficient of uniformity (Christiansen) and the reduction of potato yield for seven sprinkler spacings ( 6 x 12, 12 x 12, 12 x 15, 12 x 18, $18 \mathrm{x}$ $18,18 \times 24$ and $24 \times 24 \mathrm{~m}$ ), a multiple range analysis based on the trust interval methodology was conducted. It was observed that the larger the time of sprinkler rotation the better the uniformity coefficient under limiting spacings recommended by the manufacturer, and setting the deflection angle of $240^{\circ}$ (spring tension) as the maximum limit to avoid harmful interference on the spatial distribution of the water.
\end{abstract}

Key words: irrigation, sprinklers, spacings, adjustments, uniformity

\footnotetext{
${ }^{1}$ Professor Doutor - DER/ESALQ - USP Av. Pádua Dias 11, Piracicaba, SP. CEP 13.418-900, fone (019) 429-4217 fax $433-8134$ Email:rdcoelho@carpa.ciagri.usp.br

${ }^{2}$ Professor Livre Docente - DER/ESALQ - USP
} 


\section{INTRODUÇÃO}

A irrigação por aspersão tem contribuído acentuadamente para o aumento da área irrigada no Brasil, melhorando a produtividade das culturas e tornando mais intensiva a exploração das terras disponíveis.

Hart (1961) menciona a existência de dois tipos básicos de ensaio de distribuição espacial da água, para testes de desempenho de aspersores, em que o primeiro é um ensaio com vários aspersores operando simultaneamente, dentro do espaçamento em estudo. As precipitações obtidas nos diversos coletores da área amostrada são consideradas diretamente nos cálculos de algum tipo de coeficiente de uniformidade; o segundo tipo de ensaio consiste na coleta de dados de um único aspersor trabalhando na área; as precipitações obtidas nos coletores são posteriormente sobrepostas segundo uma lógica matricial, podendo simular diversos espaçamentos entre os emissores. $\mathrm{O}$ ensaio de um único aspersor operando é mais versátil e econômico de ser conduzido em testes de rotina.

Griffin, citado por Chen \& Wallender (1984) utilizou-se do perfil de distribuição de água de um único aspersor, obtido com coletores dispostos radialmente, para simular um ensaio de campo. A grade de coletores foi gerada por meio da rotação do perfil de distribuição em torno do eixo de rotação do aspersor e os pontos da grade não coincidentes com a real distância ao longo do ensaio radial, foram interpolados linearmente; com a grade obtida com um único aspersor, efetuaram-se as sobreposições correspondentes aos espaçamentos desejados.

Dada a variabilidade inerente aos coeficientes de uniformidade, não é possível identificar-se pequenas diferenças de desempenho de um sistema de irrigação com base em poucos testes de campo. Ensaios de laboratório (indoor) dispondo radialmente os coletores, têm sido utilizados com o objetivo de se reduzir o tempo e o espaço físico necessário na avaliação de um aspersor; este tipo de ensaio permitiu o estudo de modelos de distribuição de água de aspersores por Bean (1965).

Bilanski \& Kidder (1958) desenvolveram um trabalho célebre sobre os fatores que afetam a distribuição de água, a partir de um aspersor rotativo do tipo impacto. O estudo foi conduzido em condições de laboratório (ensaio com disposição radial de emissores) para eliminar interferências climáticas indesejáveis; dois dos fatores estudados foram o defletor-martelo e a velocidade de rotação dos emissores; com relação ao primeiro, observaram diminuição da quantidade de água precipitada no ponto de máximo alcance e, com relação ao segundo fator, pesquisando o intervalo de tempo de uma rotação compreendido entre 3 e 350 segundos, observaram redução de alcance máximo do jato de $13,12 \mathrm{~m}$ para $9,25 \mathrm{~m}$.

Coelho (1990) estudando o efeito das regulagens no desempenho do aspersor Naan modelo 233/92, constatou que o nível de tensão da mola do referido emissor altera significativamente o volume desviado do jato principal, podendo atingir valores entre 4 e $40 \%$ da vazão total. A ação do defletormartelo reduz a energia hidráulica disponível ao movimento balístico do volume d'água desviado por ocasião do impacto entre 35 e $55 \%$.

Hill \& Keller (1980) em um trabalho sobre a seleção de sistemas de irrigação visando atingir a máxima rentabilidade, utilizaram a função de distribuição da lâmina de água aplicada por um aspersor, associada a uma função de produção da cultura (lâmina aplicada x produção) para estimarem a produção total na área, de acordo com a equação (1).

$$
\mathrm{Y}=(\mathrm{A} / 100) \sum_{\mathrm{i}=1}^{\mathrm{m}} \mathrm{Pi} \mathrm{y}(\mathrm{di})
$$

em que :

Y - produção total

A - área total

m - número total de incrementos de área

di - altura de lâmina aplicada na área $i$

y - função de produção em relação à lâmina total aplicada

$\mathrm{Pi}$ - porcentagem da área que recebeu a altura de lâmina considerada

Duarte (1989) pesquisando a cultura da batata irrigada, apresentou a seguinte função de produção (Y - t.ha $\left.{ }^{-1}\right)$ com base na lâmina d'água aplicada (hi - mm):

$$
\text { Y Batata }=-28,31978+0,1818 \text { hi }-0,00014517 h^{2}
$$

Visando contribuir para uma utilização mais eficiente dos equipamentos comerciais de irrigação, este trabalho tem como objetivo estudar a influência da regulagem do aspersor na uniformidade de aplicação da água, no que diz respeito ao efeito da velocidade de giro, da tensão da mola (período de oscilação do defletor-martelo) e da pressão de operação, na distribuição espacial da lâmina aplicada e na produtividade da cultura da batata.

\section{MATERIAL E MÉTODOS}

Nos ensaios de distribuição espacial de água foram ensaiados três aspersores Naan, modelo 233/92, com o bocal intermediário de 4,4 x 2,5mm, totalizando 180 ensaios (duração de cada ensaio $=1$ hora). $\mathrm{O}$ fabricante deste aspersor recomenda sua instalação nos espaçamentos de 6 × 12, 12 × 12, 12 × 15 ou 12 x 18m; nenhuma especificação é apresentada para o tempo de rotação do emissor, tampouco para o limite máximo de tensão da mola acoplada ao defletor-martelo.

Cada emissor analisado foi submetido a 60 ensaios diferentes, combinando-se as pressões de 200,300 e $400 \mathrm{kPa}$, com tempo total de rotação do aspersor de 7, 15, 30, 60 e 90 segundos e tensão da mola nos níveis $0,1,4$ e 8 (ângulos de deflexão $0^{\circ}, 60^{\circ}, 240^{\circ}$ e $480^{\circ}$ ).

Não é possível realizar-se tal combinação de variáveis em um único aspersor operando de maneira tradicional, pois a velocidade de rotação é diretamente correlacionada ao nível de tensão na mola; para tanto, foi necessário o desenvolvimento de uma cúpula especial, dotada de uma série de recursos operacionais, apresentada na Figura 1, na qual se destaca a
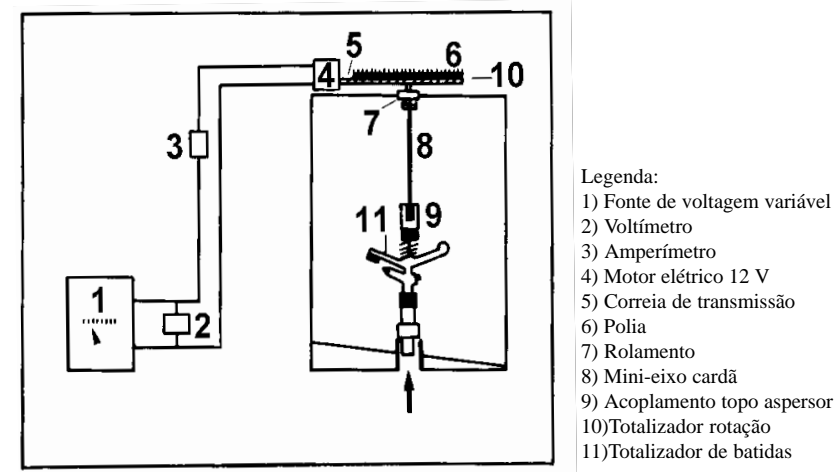

Figura 1. Representação esquemática da cúpula idealizada para controle de rotação dos aspersores ensaiados 
atuação de um motor elétrico com rotação variável conectado ao topo do aspersor, através de um mini-eixo cardã, de maneira a ser possível controlar a velocidade de giro do emissor, independente do nível de tensão da mola.

As dimensões externas da cúpula idealizada eram: $0,30 \mathrm{~m}$ de largura, $0,30 \mathrm{~m}$ de comprimento e $0,38 \mathrm{~m}$ de altura, com abertura para saída do jato na dimensão de $6 \mathrm{~cm}$ de largura $\mathrm{x}$ $25 \mathrm{~cm}$ de altura, apresentando direcionador de fluxo e anteparos internos de dissipação de energia, visando evitar o vazamento de jatos indesejáveis para fora da cúpula. Diferentes vistas da cúpula de madeira impermeabilizada encontram-se esquematizadas na Figura 2.

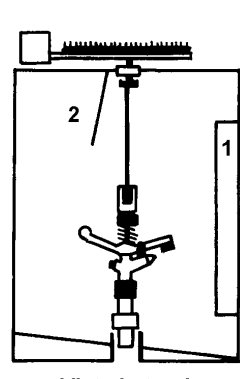

Vista Lateral

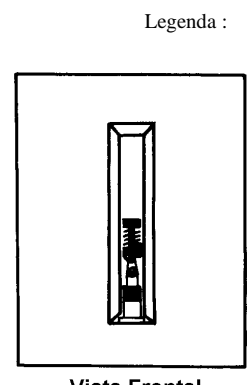

Vista Frontal
1 - Direcionador de fluxo

2 - Dissipador de energia

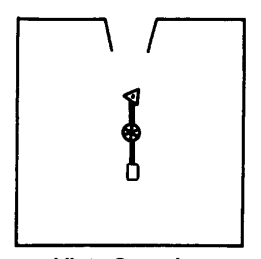

Vista Superior
Figura 2. Esquema ilustrativo das diferentes vistas da cúpula idealizada para ensaio do aspersor no interior do laboratório de irrigação

Por convenção adotou-se, como tensão 1, a menor tensão da mola que permite com que o defletor-martelo trabalhe nas condições normais de operação. As demais tensões foram provocadas por deflexões de $60^{\circ}$ do conjunto, que é o mínimo intervalo de regulagem disponível, tendo resultado as seguintes tensões: tensão $0=$ defletor posicionado fora da ação do jato, tensão $1=60^{\circ}$, tensão $2=120^{\circ}$, tensão $4=240^{\circ}$ e tensão $8=480^{\circ}$.

O ensaio de distribuição espacial de água foi desenvolvido com base na disposição radial de coletores na área de ensaio, de acordo com o esquema apresentado na Figura 3 e conduzido no interior do Laboratório de Irrigação da ESALQ (Escola Superior de Agricultura Luiz de Queiroz) de modo a evitar a influência de ventos e evaporação diferenciada das lâminas coletadas. O espaço livre disponível para a formação do jato d'água no laboratório constituía-se de uma área plana com piso de concreto, apresentando dimensões de $20 \times 5 \mathrm{~m}$ e pé direito de $6 \mathrm{~m}$. Na Figura 3 observa-se o posicionamento da linha secundária de coletores, que objetivou mensurar o perfil de distribuição do jato de água desviado pela ação do defletor-martelo.

Por uma questão de geometria, o jato d'água desviado do bocal principal sai pela janela de abertura da cúpula, com uma trajetória deslocada de aproximadamente $25^{\circ}$. A ausência da linha secundária é admitida em ensaios radiais somente quando o aspersor não ficar abrigado dentro de uma cúpula no transcorrer do teste.

Fazendo-se a revolução completa do perfil coletado simulou-se, em computador e através de um programa desenvolvido em linguagem "basic", uma grade de coletores com quadrículas de $0,5 \times 0,5 \mathrm{~m}$, considerando-se o aspersor localizado no centro da quadrícula central, obtendo-se a matriz de 64 x 64 elementos (4.100 coletores). Os coletores simulados em posições não coincidentes com os coletores do perfil coletado, tinham altura de precipitação estimada por meio de interpolação linear entre os valores dos dois coletores mais próximo.

Com base na malha espacial de um único aspersor, simularam-se os valores de lâminas precipitadas em diferentes espaçamentos entre aspersores no campo: 6 x 12, 12 x 12, 12 x 15,12 x 18, 18 x 18, 18 x 24 e 24 x 24m. Com os valores simulados das lâminas sobrepostas, foi possível, utilizando-se a metodologia proposta por Hill \& Keller (1980) e com base na função de produção da batata (Duarte 1989) estimar a produtividade da cultura e o coeficiente de uniformidade de Christiansen (CUC) para cada espaçamento analisado.

A simulação da produtividade baseou-se em duas premissas básicas: 1) em não ocorrer redistribuição horizontal de umidade significativa no solo entre as quadrículas adjacentes da malha simulada e 2) a quadrícula da grade que recebeu a lâmina média sobreposta foi a quadrícula de maior produtividade (eficiência de armazenamento 100 \%). Com relação à primeira premissa, a função de produção proposta por Duarte (1989) foi obtida com base na lâmina aplicada por um aspersor, mensurada sobre a superfície do solo (coletores); desta maneira, qualquer redistribuição horizontal de água já se encontra embutida na própria função de produção.

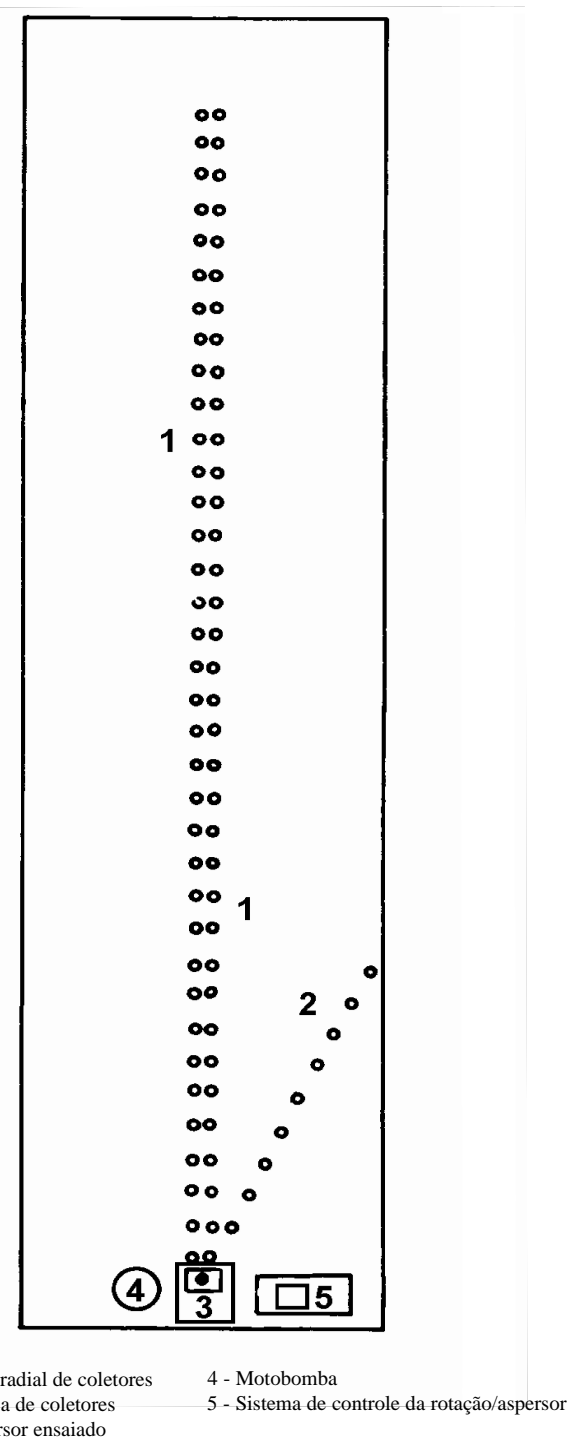

Figura 3. Distribuição radial de coletores na área de ensaio do laboratório de irrigação 


\section{RESULTADOS E DISCUSSÃO}

Com os valores médios (3 repetições) dos coeficientes de uniformidade de Christiansen (CUC) obtidos nas 60 combinações analisadas e nos 7 espaçamentos simulados, efetuou-se uma análise estatística preliminar e se verificou que os dados obtidos de CUC seguiam um modelo de distribuição normal, havendo homogeneidade de variância no ensaio fatorial realizado.

Observam-se, na Tabela 1, os resultados obtidos na análise de variância conjunta para todos os espaçamentos analisados; por esta análise concluiu-se que todos os fatores estudados (pressão, velocidade de rotação, tensão da mola e espaçamento entre aspersores) e as interações duplas entre fatores, foram significativos a nível de $1 \%$ de probabilidade.

Tabela 1. Resultados da análise de variância conjunta para todos os fatores estudados

\begin{tabular}{|c|c|c|c|c|c|}
\hline C.V. & GL & S.Q. & Q.M. & Valor $\mathrm{F}$ & $P>F$ \\
\hline Espaçamento (E) & 6 & 6,441 & 1,073 & 764,320 & 0,00001 \\
\hline Pressão (P) & 2 & 0,304 & 0,152 & 108,396 & 0,00001 \\
\hline Velocidade (V) & 4 & 0,510 & 0,127 & 90,943 & 0,00001 \\
\hline Tensão Mola (T) & 3 & 0,440 & 0,146 & 104,615 & 0,00001 \\
\hline (E) $x(P)$ & 12 & 0,055 & 0,004 & 3,285 & 0,00301 \\
\hline (E) $x(V)$ & 24 & 0,685 & 0,028 & 20,332 & 0,00001 \\
\hline (E) $\mathrm{x}(\mathrm{T})$ & 18 & 0,054 & 0,030 & 2,160 & 0,00459 \\
\hline$(\mathrm{P}) \times(\mathrm{V})$ & 8 & 0,050 & 0,006 & 4,473 & 0,00011 \\
\hline (P) $\mathrm{x}(\mathrm{T})$ & 6 & 0,053 & 0,008 & 6,385 & 0,00002 \\
\hline (V) $\mathrm{x}(\mathrm{T})$ & 12 & 0,198 & 0,016 & 11,787 & 0,00001 \\
\hline Resíduo & 224 & 0,455 & 0,001 & & \\
\hline TOTAL & 419 & 9,250 & & & \\
\hline
\end{tabular}

Simultaneamente, realizou-se uma análise multifatorial de variância, pelo método do intervalo de confiança a $95 \%$ de probabilidade, para simplificar a compreensão dos resultados obtidos; já na Tabela 2 encontram-se os resultados obtidos para os coeficientes de uniformidade (CUC) obtidos para 6 dos 7 espaçamentos enfocados neste estudo. Os valores de CUC apresentados são média dos ensaios realizados, dentro de cada nível de fator analisado, dispostos de maneira crescente, para cada espaçamento considerado.

$\mathrm{Na}$ Tabela 3 é apresentada a estimativa da porcentagem de queda $(\% \mathrm{Q})$ na produção potencial da batata $\left(28,59\right.$ t.ha $\left.^{-1}\right)$ para as diferentes combinações de pressão, tempo de rotação - TR (s) e nível de tensão da mola - T, para o aspersor Naan, modelo 233/92 (bocal 4,4 x 2,5mm) no espaçamento entre aspersores de 12 x 18m. Observa-se, nesta Tabela, que a medida em que a pressão diminui, aumenta a influência da velocidade de rotação e da tensão da mola na queda da estimativa de produção da batata; nota-se, também, uma acentuada influência dos níveis de tensão 4 e 8 da mola, pois os maiores valores de queda na produção potencial da batata estão associados a esses valores de tensão; com relação ao tempo de rotação, tem-se uma relativa influência dos tempos de 7 e 15 segundos nos maiores valores de porcentagem de queda na produção da batata; por outro lado, observou-se, para os níveis de tensão de 0 e 1 e tempos de giro de 30, 60 e 90 segundos, a menor interferência na produção da cultura, além de se observar, também, para os níveis de tensão de 0 e 1 e tempos de rotação de 30,60 e 90 segundos, a menor interferência na produção da batata. Como o nível de tensão 0 não se aplica na prática, conclui-se que o nível de tensão 1 da mola e a velocidade de giro entre 30 e 60 segundos são as regulagens mais apropriadas para operação do aspersor Naan modelo 233/92, no espaçamento de $12 \times 18 \mathrm{~m}$.

Tabela 2. Análise multifatorial de variância (método do intervalo de confiança a $95 \%$ de probabilidade), dos coeficientes de uniformidade de Christiansen (CUC), dentro de cada nível de fator estudado (pressão, tempo de rotação e tensão da mola do aspersor) para diferentes espaçamentos entre emissores

\begin{tabular}{|c|c|c|c|c|c|c|c|c|c|c|c|c|c|c|c|c|c|c|}
\hline & \multicolumn{3}{|c|}{$6 \times 12 m$} & \multicolumn{3}{|c|}{$12 \times 12 \mathrm{~m}$} & \multicolumn{3}{|c|}{$12 \times 18 \mathrm{~m}$} & \multicolumn{3}{|c|}{$18 \times 18 \mathrm{~m}$} & \multicolumn{3}{|c|}{$18 \times 24 \mathrm{~m}$} & \multicolumn{3}{|c|}{$24 \times 24 \mathrm{~m}$} \\
\hline & \multicolumn{3}{|c|}{ CUC } & \multicolumn{3}{|c|}{ CUC } & \multicolumn{3}{|c|}{ CUC } & \multicolumn{3}{|c|}{ CUC } & \multicolumn{3}{|c|}{ CUC } & \multicolumn{3}{|c|}{ CUC } \\
\hline & 40 & 0.92 & $\mathrm{a}$ & 40 & 0.88 & $\mathrm{a}$ & 40 & 0.82 & $\mathrm{a}$ & 30 & 0.78 & $\mathrm{a}$ & 30 & 0.64 & $\mathrm{a}$ & 30 & 0.55 & $\mathrm{a}$ \\
\hline PRESSÃO & 30 & 0.89 & $\mathrm{~b}$ & 30 & 0.85 & $\mathrm{~b}$ & 30 & 0.81 & $\mathrm{a}$ & 40 & 0.77 & $\mathrm{a}$ & 40 & 0.61 & $a b$ & 40 & 0.52 & $\mathrm{a}$ \\
\hline$(\mathrm{x} 10 \mathrm{kPa})$ & 20 & 0.86 & $\mathrm{c}$ & 20 & 0.79 & $\mathrm{c}$ & 20 & 0.73 & $\mathrm{~b}$ & 20 & 0.73 & $\mathrm{a}$ & 20 & 0.58 & $\mathrm{~b}$ & 20 & 0.49 & $\mathrm{a}$ \\
\hline & 15 & 0.91 & $\mathrm{a}$ & 30 & 0.87 & $\mathrm{a}$ & 90 & 0.81 & $\mathrm{a}$ & 30 & 0.81 & $\mathrm{a}$ & 60 & 0.70 & $\mathrm{a}$ & 90 & 0.61 & $\mathrm{a}$ \\
\hline TEMPO & 30 & 0.91 & $\mathrm{a}$ & 15 & 0.87 & $\mathrm{a}$ & 60 & 0.81 & a & 60 & 0.80 & $a b$ & 90 & 0.69 & a & 60 & 0.59 & $\mathrm{a}$ \\
\hline DE & 7 & 0.89 & $a b$ & 7 & 0.84 & $a b$ & 30 & 0.80 & $\mathrm{a}$ & 90 & 0.76 & $a b$ & 30 & 0.62 & $\mathrm{~b}$ & 30 & 0.57 & $\mathrm{a}$ \\
\hline ROTAÇÃO & 60 & 0.87 & $\mathrm{~b}$ & 60 & 0.82 & $\mathrm{~b}$ & 15 & 0.78 & $\mathrm{~b}$ & 15 & 0.75 & $\mathrm{~b}$ & 15 & 0.56 & $\mathrm{~b}$ & 15 & 0.49 & $\mathrm{~b}$ \\
\hline (segundos) & 90 & 0.87 & $\mathrm{~b}$ & 90 & 0.80 & $\mathrm{~b}$ & 7 & 0.73 & $\mathrm{~b}$ & 7 & 0.68 & $\mathrm{c}$ & 7 & 0.49 & $\mathrm{c}$ & 7 & 0.35 & $\mathrm{c}$ \\
\hline & 1 & 0.90 & $\mathrm{a}$ & 1 & 0.86 & $\mathrm{a}$ & 1 & 0.82 & $\mathrm{a}$ & 1 & 0.79 & $\mathrm{a}$ & 1 & 0.65 & $\mathrm{a}$ & 4 & 0.56 & $\mathrm{a}$ \\
\hline ENSÃO DA & 0 & 0.90 & $\mathrm{a}$ & 0 & 0.85 & $\mathrm{a}$ & 0 & 0.81 & $\mathrm{a}$ & 0 & 0.79 & $\mathrm{a}$ & 4 & 0.63 & $\mathrm{a}$ & 1 & 0.54 & $\mathrm{a}$ \\
\hline MOLA & 4 & 0.89 & $a b$ & 4 & 0.84 & $a b$ & 4 & 0.79 & $\mathrm{a}$ & 4 & 0.77 & $\mathrm{a}$ & 0 & 0.62 & $\mathrm{a}$ & 8 & 0.53 & $\mathrm{a}$ \\
\hline ( níveis) & 8 & 0.87 & $\mathrm{~b}$ & 8 & 0.81 & $\mathrm{~b}$ & 8 & 0.73 & $\mathrm{~b}$ & 8 & 0.69 & $\mathrm{~b}$ & 8 & 0.54 & $\mathrm{~b}$ & 0 & 0.45 & $\mathrm{~b}$ \\
\hline
\end{tabular}

Obs 1. Dentro de cada espaçamento estudado são apresentados os valores de CUC, dispostos em ordem decrescente, de acordo com o nível do fator analisado (valores em itálico à esquerda dos valores de CUC) Obs 2 . Ao lado direito dos valores de CUC, em disposição vertical, encontram-se as letras: $a, b$ e $c$ que indicam, para cada fator analisado, valores de média estatisticamente semelhantes pela metodologia do intervalo de confiança a $95 \%$ de probabilidade 
Tabela 3. Estimativa da porcentagem de queda (\%Q) na produção potencial da batata $\left(28,59\right.$ t.ha $\left.^{-1}\right)$ para as diferentes combinações de pressão, tempo de rotação - TR (s) e nível de tensão da mola - T, para o aspersor Naan, modelo 233/92 (bocal 4,4 x $2,5 \mathrm{~mm})$ no espaçamento entre aspersores de $12 \times 18 \mathrm{~m}$

\begin{tabular}{|c|c|c|c|c|c|c|c|c|}
\hline \multicolumn{9}{|c|}{ Pressão } \\
\hline \multicolumn{3}{|c|}{$200 \mathrm{kPa}$} & \multicolumn{3}{|c|}{$300 \mathrm{kPa}$} & \multicolumn{3}{|c|}{$400 \mathrm{kPa}$} \\
\hline TR & $\mathrm{T}$ & $\% \mathrm{Q}$ & TR & $\mathrm{T}$ & $\% \mathrm{Q}$ & $\overline{T R}$ & $\mathrm{~T}$ & $\% \mathrm{Q}$ \\
\hline 7 & 8 & 41,39 & 7 & 8 & 22,51 & 7 & 8 & 28,89 \\
\hline 30 & 8 & 26,35 & 15 & 8 & 21,00 & 7 & 0 & 18,97 \\
\hline 15 & 8 & 25,39 & 30 & 8 & 18,21 & 15 & 8 & 18,13 \\
\hline 30 & 0 & 22,26 & 7 & 4 & 15,03 & 15 & 4 & 14,16 \\
\hline 60 & 8 & 22,17 & 60 & 8 & 12,92 & 7 & 4 & 12,41 \\
\hline 60 & 4 & 21,70 & 15 & 4 & 11,68 & 60 & 8 & 11,59 \\
\hline 90 & 4 & 21,49 & 7 & 0 & 11,18 & 7 & 1 & 11,57 \\
\hline 60 & 0 & 19,58 & 7 & 1 & 10,39 & 15 & 1 & 10,24 \\
\hline 30 & 4 & 19,45 & 30 & 4 & 10,19 & 90 & 0 & 7,46 \\
\hline 7 & 4 & 18,44 & 60 & 4 & 9,42 & 30 & 8 & 7,43 \\
\hline 15 & 4 & 17,87 & 90 & 8 & 8,59 & 90 & 8 & 6,68 \\
\hline 90 & 8 & 17,50 & 90 & 4 & 8,49 & 60 & 4 & 6,67 \\
\hline 90 & 1 & 15,94 & 90 & 1 & 8,19 & 30 & 4 & 5,80 \\
\hline 60 & 1 & 15,15 & 30 & 0 & 7,41 & 90 & 1 & 5,34 \\
\hline 90 & 0 & 14,64 & 15 & 1 & 7,11 & 30 & 1 & 4,75 \\
\hline 15 & 0 & 13,01 & 60 & 0 & 6,49 & 15 & 0 & 4,45 \\
\hline 7 & 0 & 12,16 & 30 & 1 & 5,45 & 60 & 0 & 4,33 \\
\hline 15 & 1 & 11,90 & 60 & 1 & 5,78 & 60 & 1 & 4,19 \\
\hline 30 & 1 & 10,28 & 90 & 0 & 5,76 & 90 & 4 & 4,15 \\
\hline 7 & 1 & 10,24 & 15 & 0 & 5,46 & 30 & 0 & 4,04 \\
\hline
\end{tabular}

Observa-se, na Figura 4, a correlação gráfica entre os valores de coeficientes de uniformidade (CUC) e a redução porcentual de produção da cultura de batata, para todas as combinações analisadas entre pressão, tempo de giro, tensão da mola e espaçamento entre emissores. Observa-se que não existe uma função unívoca entre CUC e porcentagem de queda (\%Q) na produção potencial da batata, principalmente para valores de CUC menores que $80 \%$, ou seja, um único valor de coeficiente de uniformidade de Christiansen (CUC) pode representar duas ou mais distribuições espaciais de água, de que resultam diferentes estimativas de produtividades de uma mesma cultura.

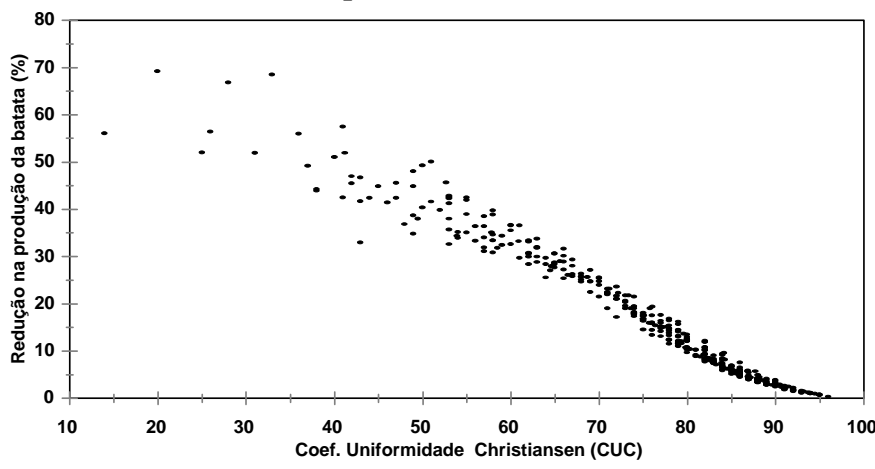

Figura 4. Relação entre os valores de coeficientes de uniformidade de Christiansen (CUC) e porcentagem de queda na produção potencial da cultura da batata

\section{CONCLUSÕES}

De acordo com os resultados dos ensaios e simulações realizadas com o aspersor Naan, modelo 233/92, pode-se concluir que:

1. A pressão de operação do aspersor interfere na distribuição de água e na estimativa da produtividade da cultura de batata. Nos menores espaçamentos entre aspersores $(6 \times 12$ e 12 x $12 \mathrm{~m}$ ) as maiores pressões (300 e $400 \mathrm{kPa}$ ) apresentam maiores valores de coeficientes de uniformidade (CUC) enquanto nos demais espaçamentos ( 12 x 18 a $18 \times 18 \mathrm{~m})$ as pressões de 200 , 300 e 400kPa não diferem significativamente quanto aos valores de CUC apresentados

2. A velocidade de rotação do aspersor influencia a distribuição espacial de água e a estimativa de produtividade da cultura de batata, sendo que a velocidade ideal de giro depende do espaçamento entre aspersores. No menor espaçamento analisado $(6 \times 12 \mathrm{~m})$ maior velocidade de rotação $(7$ a 30 segundos) é favorável; em espaçamentos intermediários (12 x $15 \mathrm{~m})$ a velocidade de rotação entre 7 e 90 segundos não interfere significativamente; já nos maiores espaçamentos (18 x 18m) o ideal é uma velocidade de rotação mais reduzida (30 a 90 segundos).

3. O nível 4 de tensão da mola $\left(240^{\circ}\right)$ acoplada ao defletormartelo pode ser estabelecido como o limite máximo ideal de funcionamento do referido aspersor.

4. Um único valor de coeficiente de uniformidade de Christiansen (CUC) pode representar duas ou mais distribuições espaciais de água, que resultam em diferentes produtividades da cultura de batata.

\section{REFERÊNCIAS BIBLIOGRÁFICAS}

BEAN, A.G.M. Water distribution by irrigation sprinkler. Journal of Agricultural Engineering Research, Cambridge, v.10, p.314-321, 1965.

BILANSKI, W.K.; KIDDER, E.H. Factors that affect the distribution of water from a medium pressure rotary irrigation sprinkle. Transactions of the ASAE, St. Joseph, v.1, n.1, p.10-28, 1958.

CHEN, D.; WALLENDER, W.W. Economic sprinkler selection spacing and orientation. Transactions of the ASAE, St. Joseph, v.27, n.3, p.737-743, 1984.

COELHO, R.D. Regulagem de aspersores e seus efeitos sobre a uniformidade de aplicação de água e produtividade das culturas. Piracicaba: ESALQ. 1990, 141p. Dissertação Mestrado

DUARTE, S.N. Efeitos do horário e da lâmina de irrigação na cultura da batata (Solanum tuberosum L.) . Piracicaba: ESALQ. 1989, 148p. (Dissertação Mestrado)

HART, W.E. Overhead irrigation pattern parameters. Transactions of the ASAE, St. Joseph, v.42, n.7, 354-355, 1961.

HILL, R.W.; KELLER, J. Irrigation selection for maximum crop profit. Transactions of the ASAE, St. Joseph, v.23, n.2, p.366-372, 1980 . 